\title{
A High Spectral Efficient Non-Binary TCM Scheme-Based Novel Decoding Algorithm for 4G Systems
}

\author{
Riyadh A. Al-Hilali ${ }^{1}$, Raad H. Thaher ${ }^{1}$, Abdulkareem S. Abdallah ${ }^{2}$ \\ ${ }^{1}$ College of Engineering, Al-Mustansiriya University, Baghdad, Iraq \\ ${ }^{2}$ College of Engineering, Basrah University, Basrah, Iraq \\ Email: Riyadh_alhilali@yahoo.com,raadthaher@yahoo.com, kareem134@yahoo.com
}

Received July 1, 2013; revised July 30, 2013; accepted August 10, 2013

Copyright (C) 2013 Riyadh A. Al-Hilali et al. This is an open access article distributed under the Creative Commons Attribution License, which permits unrestricted use, distribution, and reproduction in any medium, provided the original work is properly cited.

\begin{abstract}
This paper deals with the MIMO-OFDM technique that is applied to the fourth generation (4G) of the wireless communication systems, this technique can provide high data rate transmission without increasing transmit power and expanding bandwidth, it can also efficiently use space resources and has a bright future. It presents the channel coding assisted STBC-OFDM systems, and employs the Coded Modulation techniques (CM), since the signal bandwidth available for wireless communications is limited. The proposed system deals with Non-binary error control coding of the TCM-aided STBC-OFDM scheme for transmissions over the Rayleigh channel. A new non-binary decoding method, Yaletharatalhussein decoding algorithm, is designed and implemented for decoding non-binary convolutional codes, which is based on the trellis diagram representing the convolutional encoder. Yaletharatalhussein decoding algorithm outperforms the Viterbi algorithm and other algorithms in its simplicity, very small computational complexity, decoding reliability for high states TCM codes that are suitable for Fourth-Generation (4G), decreasing errors with increasing word length, and easy to implement with real-time applications. The simulation results show that the performance of the non-binary TCM-based Yaletharatalhussein decoding algorithm-assisted STBC-OFDM scheme outperforms the binary and non-binary decoding methods.
\end{abstract}

Keywords: Convolutional Codes; Trellis Code Modulation(TCM); Non-Binary Error Correcting Codes; Groups; Rings of Integers; OFDM; MIMO; MIMO-OFDM; STBC

\section{Introduction}

The fourth generation of wireless communications refers to a collection of technologies and standards that will find their way into a range of new ubiquitous computing and connections systems. 4G offers the promise of allowing users to connect to the Internet and one another through a variety of devices and standards anytime, anywhere, and at a wide range of speeds, from narrowband to broadband $[1,2]$. The main key features of $4 \mathrm{G}$ are receiving a large volume of information, data, pictures, video and so on. As a key building block of next-generation wireless communication systems, MIMOs are capable of supporting significantly higher data rates than the Universal Mobile Telecommunications System (UMTS) and the High-Speed Downlink Packet Access (HSDPA) based $3 \mathrm{G}$ networks [3]. A MIMO system is capable of exploiting transmitter and receiver diversity, hence maintaining reliable communications. To overcome a multi- path-fading environment with low complexity and to achieve wireless broadband multimedia communication systems (WBMCS), the orthogonal frequency-division multiplexing (OFDM) transmission scheme is employed [4]. OFDM is one of the applications of a parallel-datatransmission scheme, which reduces the influence of multipath fading and makes complex equalizers unnecessary. Advances in coding, such as turbo [5] and low density parity check codes [6], made it feasible to approach the Shannon capacity limit [7] in systems with a single antenna link. Significant further advances in spectral efficiency are available through increasing the number of antennas at both the transmitter and the receiver [8-10].

Further performance gains can be achieved by using non-binary codes in the coded modulation scheme, but with an increase in the decoding complexity [11]. Nonbinary codes are the most commonly used error-correcting codes and can be found in optical and magnetic stor- 
age, high-speed modems and wireless communications. J. Jayakumari [12], analyzed the performance of MIMOOFDM system employing Quadrature Amplitude Modulation (QAM) in Rayleigh fading channel, since the use of multiple antennas at both ends of a wireless link (multiple-input multiple output (MIMO) technology) has recently been demonstrated to have the potential of achieving extraordinary data rates. Orthogonal frequency division multiplexing (OFDM) significantly reduces receiver complexity in wireless broadband systems. The use of MIMO technology in combination with OFDM, i.e., MIMO-OFDM therefore seems to be an attractive solution for future broadband wireless systems.

L. Hanzo et al. [13] presented that the CM-assisted STBC schemes were found to significantly improve the system's achievable performance. Furthermore, the TTCMSTBC concatenated scheme was observed to give the best performance among all the CM-STBC concatenated schemes. Also, the TTCM-assisted $G_{2}$ coded OFDM scheme gives a better performance than the LDPC-assisted $G_{2}$ coded OFDM scheme. Furthermore, in the context of the achievable coding gain versus complexity performance, it is found that the TTCM-assisted schemes are capable of achieving higher coding gains in the relatively high-complexity range, than the LDPC-assisted candidate schemes.

\section{OFDM, MIMO, and MIMO-OFDM}

The principle of multi-carrier transmission is to convert a serial high rate data stream on to multiple parallel low rate sub-streams. Each sub-stream is modulated on another sub-carrier. Since the symbol rate on each subcarrier is much less than the initial serial data symbol rate, the effects of delay spread, i.e. ISI, significantly decrease, reducing the complexity of the equalizer. OFDM is a low complex technique used to modulate multiple sub-carriers efficiently by using digital signal processing [14-17]. An important design goal for a multi-carrier transmission scheme based on OFDM in a mobile radio channel is that the channel can be considered as time-invariant during one OFDM symbol and that fading per sub-channel can be considered as flat.

As conventional methods like using more bandwidth or higher order modulation types are limited, new methods of using thetransmission channel have to be used. Multiple antenna systems (Multiple Input, Multiple Output-MIMO) give a significantenhancement to data rate and channel capacity. A MIMO system employs multiple transmitter and receiver antennas for delivering parallel data streams, as illustrated in Figure 1. Since the information is transmitted through different paths, a MIMO system is capable of exploiting transmitter and receiver diversity, hence maintaining reliable communications.

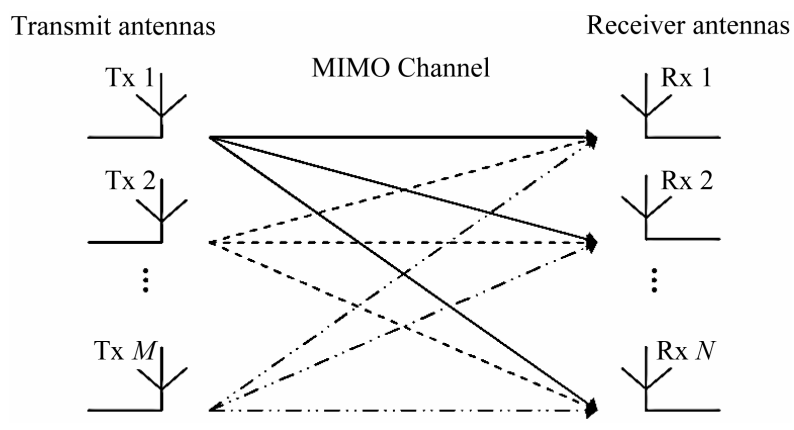

Figure 1. A MIMO system.

As compared with Single-Input, Single-Output (SISO) systems, the most significant advantages of MIMO systems are; a significant increase of both the system's capacity and spectral efficiency, and dramatic reduction of the effects of fading due to the increased diversity.

The quality of a wireless link can be described by three basic parameters, namely the transmission rate, the transmission range and the transmission reliability. Conventionally, the transmission rate may be increased by reducing the transmission range and reliability. By contrast, the transmission range may be extended at the cost of a lower transmission rate and reliability, while the transmission reliability may be improved by reducing the transmission rate and range [18].

However, with the advent of MIMO-assisted OFDM systems, the above-mentioned three parameters may be simultaneously improved. Initial field tests of broadband wireless MIMO-OFDM communication systems have shown that an increased capacity, coverage and reliability are achievable with the aid of MIMO techniques [19].

A generic MIMO-OFDM system employing $\mathrm{K}$ orthogonal frequency-domain subcarriers and having $m_{\mathrm{t}}$ and $n_{\mathrm{r}}$ transmit and receive antennas, respectively.

Firstly, the OFDM modulation technique is capable of coping with the highly frequency-selective, time-variant channel characteristics associated with mobile wireless communication channels, while possessing a high grade of structural flexibility for exploiting the beneficial properties of MIMO architectures.

The family of space-time signal processing methods, which allow for the efficient implementation of communication systems employing MIMO architectures, is commonly referred to as smart antennas.

The schematic of a typical MIMO-OFDM system's physical layer is depicted in Figure 2. The transmitter of the MIMO-OFDM system considered is typically constituted by the encoder and modulator, generating a set of $m_{\mathrm{t}}$ complex-valued baseband time-domain signals. The modulated baseband signals are then processed in parallel.

Specifically, they are over sampled and shaped using a Nyquist filter. The resultant over sampled signals are 


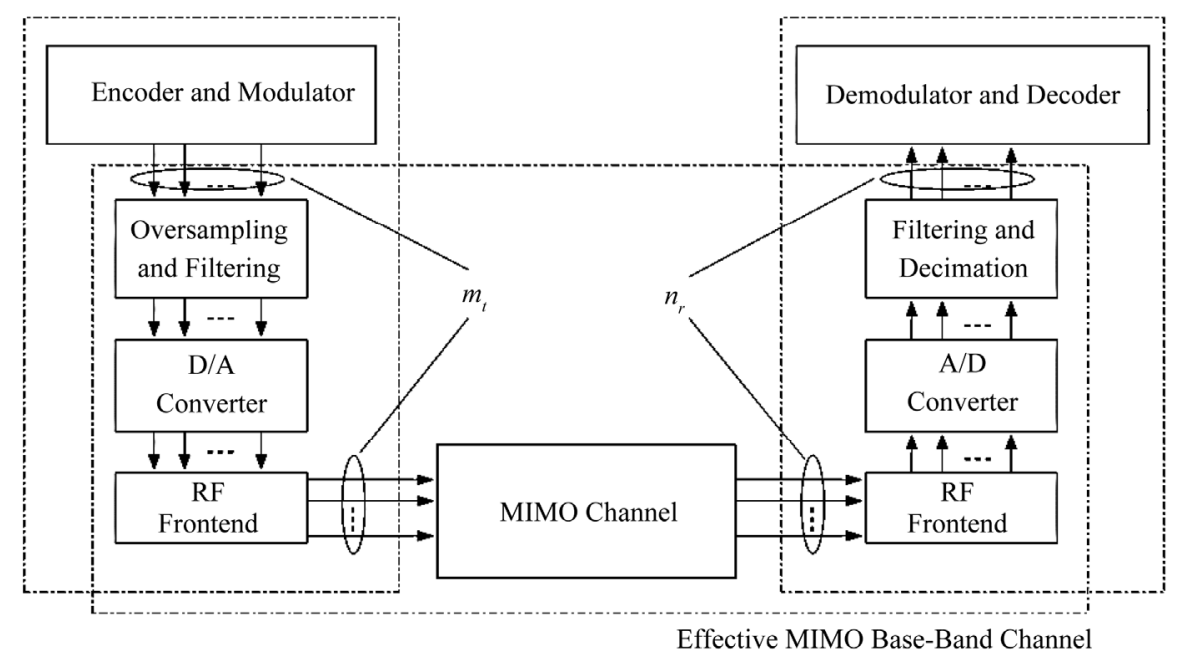

Figure 2. Schematic of a typical MIMO-OFDM system's physical layer.

then converted into an analogue passband signal using a bank of D/A convertersand upconverted to the RF band.

At the receiver side of the MIMO-OFDM transceiver, the inverse process takes place, where the set of received RF signals associated with the $n_{\mathrm{r}}$ receive antenna elements is amplified by the RF amplifier and downconverted to an intermediate-frequency passband.

The resultant passband signals are then sampled by a bank of A/D converters, downconverted to the baseband, filtered by a matched Nyquist filter and finally decimated, in order to produce a set of discrete complex-valued baseband signals. The resultant set of discrete signals is processed by the corresponding demodulator and decoder module seen in Figure 2 where the transmitted information-carrying symbols are detected.

The discrete frequency-domain model of the MIMOOFDM system is illustrated in Figure 2 may be characterized as a generalization of the Single-Input SingleOutput (SISO) case:

$$
y_{i}[n, k]=\sum_{j=1}^{m_{t}} H_{i j}[n, k] x_{j}[n, k]+w_{i}[n, k]
$$

where $n=0,1, \cdots$ and $k=0, \cdots, K-1$ are the OFDM symbol and subcarrier indices, respectively, while $y_{i}[n$, $k], x_{j}[n, k]$ and $w_{i}[n, k]$ denote the symbol received at the $i$ th receive antenna, the symbol transmitted from the $j$ th transmit antenna and the Gaussian noise sample encountered at the $i$ th receive antenna, respectively. Furthermore, $H_{i j}[n, k]$ represents the complex-valued CTF coefficient associated with the propagation link connecting the $j$ th transmit and $i$ th receive antennas at the $k$ th OFDM subcarrier and time instance $n$. The MIMO-OFDM system model described by (1) can be interpreted as the perOFDM-subcarrier vector expression of

$$
\boldsymbol{y}[n, k]=\boldsymbol{H}[n, k] \boldsymbol{X}[n, k]+\boldsymbol{W}[n, k]
$$

\section{Alamouti's $G_{2}$ STBC}

The system contains two transmitter antennas and one receiver antenna, a generic STBC is defined by an $(n \times$ $p$ )-dimensional transmission matrix $G$, where the entries of the matrix $G$ are linear combinations of the $k$ input symbols $x_{1}, x_{2}, \cdots, x_{k}$ and their conjugates. Each symbol $x_{i}(i=1, \cdots, k)$ conveys $b$ original information bits according to the relevant signal constellation that has $M=$ $2^{b}$ constellation points, and hence can be regarded as information symbols. Thus, $(k \times b)$ input bits are conveyed by each $(n \times p)$ block.

The $G_{2}$ transmission matrix can be derived in the form of [20].

$$
\left[\begin{array}{cc}
x_{1} & x_{2} \\
-\bar{x}_{2} & \bar{x}_{1}
\end{array}\right]
$$

Since there are $k=2$ input symbols, namely $x_{1}$ and $x_{2}$, the code rate of $G_{2}$ is $R=k / n=1$.

Two algorithms are widely used for decoding STBCs. The maximum likelihood (ML) decoding algorithm generates hard-decision outputs, while the Maximum-APosteriori (MAP) decoding algorithm is capable of providing soft outputs, which readily lend themselves to channel coding for achieving further performance improvements.

\subsection{Maximum-A-Posteriori Decoding}

Bauch [21] presented a simple symbol-by-symbol MAP algorithm for decoding STBCs. According to [21], the a posteriori probability of each information symbol $x_{i}(i=1, \cdots, k)$ is given by

$$
\begin{aligned}
& \ln P\left(x_{i} \mid y_{1}, y_{2}, \cdots, y_{q}\right) \\
& =\text { const }+\ln P\left(y_{1}, y_{2}, \cdots, y_{q} \mid x_{i}\right)+\ln P\left(x_{i}\right)
\end{aligned}
$$


where $\left[y_{1}^{j}, y_{2}^{j}, \cdots, y_{n}^{j}\right](j=1, \cdots, q)$ represents the received signal vector at receiver $j$ during the period span- ning from time slot 1 to time slot $n$, for the STBC $G_{2}(k=$ $2, n=2$ ) the a posteriori probabilities are given by

$$
\begin{aligned}
& \ln P\left(x_{1} \mid y_{1}, y_{2}, \cdots, y_{q}\right)=\mathrm{const}-\frac{1}{2 \sigma^{2}}\left\{\left|\left[\sum_{j=1}^{q}\left(y_{1}^{j} \bar{h}_{1, j}+\bar{y}_{2}^{j} h_{2, j}\right)\right]-x_{1}\right|^{2}+\left(\sum_{j=1}^{q} \sum_{i=1}^{2}\left|h_{i, j}\right|^{2}-1\right)\left|x_{1}\right|^{2}\right\}+\ln P\left(x_{1}\right) \\
& \ln P\left(x_{2} \mid y_{1}, y_{2}, \cdots, y_{q}\right)=\mathrm{const}-\frac{1}{2 \sigma^{2}}\left\{\left|\left[\sum_{j=1}^{q}\left(y_{1}^{j} \bar{h}_{2, j}+\bar{y}_{2}^{j} h_{1, j}\right)\right]-x_{2}\right|^{2}+\left(\sum_{j=1}^{q} \sum_{i=1}^{2}\left|h_{i, j}\right|^{2}-1\right)\left|x_{2}\right|^{2}\right\}+\ln P\left(x_{2}\right)
\end{aligned}
$$

The corresponding a posteriori probabilities of the bits (i.e. the corresponding soft outputs) using the symbol-to-bit probability conversion of

$$
\begin{aligned}
& P\left(d_{i}=0\right)=\sum_{j} P\left(x_{j} \mid y_{1}, y_{2}, \cdots, y_{q}\right), \forall x_{i}=\left(d_{1} \cdots d_{i} \cdots d_{b}\right), d_{i}=0, \\
& P\left(d_{i}=1\right)=\sum_{j} P\left(x_{j} \mid y_{1}, y_{2}, \cdots, y_{q}\right), \forall x_{i}=\left(d_{1} \cdots d_{i} \cdots d_{b}\right), d_{i}=1,
\end{aligned}
$$

where $P\left(d_{i}=0\right)$ or $P\left(d_{i}=1\right)$ represents the probability of the $i$ th bit, namely $d_{i}$, of the $b$-bit symbol being zero and one, respectively. Then the relevant soft outputs can be forwarded to the channel decoders, which will make a hard decision to finally decode the received signals.

\subsection{Channel-Coded STBC}

The MAP algorithm invoked for decoding STBCs can be exploited by concatenated channel decoders for further improving the system's performance. This paper concatenates on the STBCs with a Turbo Convolutional (TC) code $[4,22]$. The STBCs can also be concatenated with a range of other channel codes, such as Convolutional Codes (CCs), Turbo Bose-Chaudhuri-Hocquenghem (TBCH) codes (a class of FEC codes), etc. The best scheme found was the half-rate TC $(2,1,4)$ code in conjunction with the STBC $G_{2}$. TTCM [23] is a more recent joint coding and modulation scheme which has a structure similar to that of the family of binary turbo codes,

\section{Non-Binary TCM}

Convolutional codes and TCM codes are based on rings of integers modulo-M. Due to the similarities between M-PSK signal sets and the algebraic structure of rings of integers modulo-M, modulo-M ring-TCM codes are the natural linear codes for M-PSK modulation.

\section{TCM Based on Rings of Integers}

The general structure of a ring-TCM encoder suitable for M-PSK modulation, assuming that $m$ information bits are transmitted per baud, with $M=2^{m+1}$, is shown in Figure 3. This ring-TCM encoder works as follows [24]:

- First, $m+1$ information bits, $b_{i}$, are mapped into a
modulo-M symbol, $a_{j}$, according to a mapping function $f$ (for instance, $f$ can be a Gray mapping function).

- Next, $m$ modulo-M $a_{j}$ symbols are introduced into a linear multi-level convolutional encoder (MCE), which generates $m+1$ modulo-M coded symbols, $x_{k}$.

- Finally, each one of these coded symbols $x_{k}$ is associated with a signal of the M-PSK signal set and is sent to the channel.

As a total of $m+1$ modulo-M coded symbols $x_{k}$ are transmitted per single trellis branch, ring-TCM codes can be considered as $2(m+1)$-dimensional TCM codes.

\section{Rings of Integers}

If the two binary operations "+" and "." are allowed then a ring can be defined. A ring must have the following conditions; associativity, distributivity, and commutativity under addition. The ring is called a commutative ring if it also has commutativity under multiplication. If the ring has a multiplicative identity 1 then it is called a ring with identity. An example of a ring is the ring of integers $\mathbb{Z}_{q}$ under modulo-q addition and multiplication, where $\mathrm{q}$ is the cardinality of the ring. For example, $\mathbb{Z}_{4}$ is defined as $\{0,1,2,3\}$.

It is easy to see that the elements obey the three definitions of a ring. Also, all the elements commute under multiplication and the multiplicative identity element 1 is present, meaning that $\mathbb{Z}_{4}$ is a commutative ring with identity. Tables 1 and 2 show the addition and multiplication tables respectively of the ring of integers $\mathbb{Z}_{4}=\{0,1,2,3\} \quad[25]$.

The set of all polynomials with coefficients defined in $\mathbb{Z}_{q}$ forms a ring under the addition and multiplication operations. 


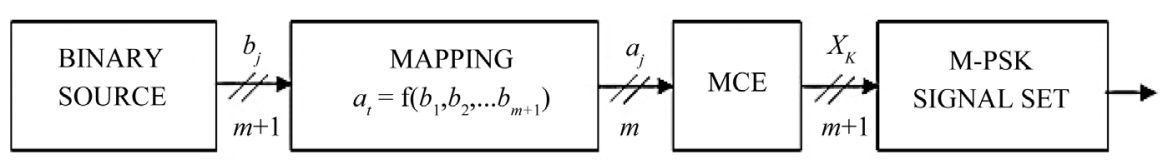

Figure 3. General structure of a ring-TCM encoder suitable for M-PSK modulation.

Table 1. Addition table for $\mathbb{Z}_{4}$.

\begin{tabular}{lllll}
\hline+ & $\mathbf{0}$ & $\mathbf{1}$ & $\mathbf{2}$ & $\mathbf{3}$ \\
\hline $\mathbf{0}$ & 0 & 1 & 2 & 3 \\
$\mathbf{1}$ & 1 & 2 & 3 & 0 \\
$\mathbf{2}$ & 2 & 3 & 0 & 1 \\
$\mathbf{3}$ & 3 & 0 & 1 & 2 \\
\hline
\end{tabular}

Table 2. Multiplication table for $\mathbb{Z}_{4}$.

\begin{tabular}{lllll}
\hline $\boldsymbol{1}$ & $\mathbf{0}$ & $\mathbf{1}$ & $\mathbf{2}$ & $\mathbf{3}$ \\
\hline $\mathbf{0}$ & 0 & 0 & 0 & 0 \\
$\mathbf{1}$ & 0 & 1 & 2 & 3 \\
$\mathbf{2}$ & 0 & 2 & 0 & 2 \\
$\mathbf{3}$ & 0 & 3 & 2 & 1 \\
\hline
\end{tabular}

\section{A 4-State Ring-TCM Code Defined over 4}

A good 4-state ring-TCM code over $\mathbb{Z}_{4}$ is shown in Figure 4 and this would be used in this research work [24].

Where each of the input and output of Figure 4 represent the elements of the ring of integers modulo-4, $\mathbb{Z}_{4}=\{0,1,2,3\}$, and all addition arithmetic operations satisfy the properties of the ring of integers modulo-4 addition. The delay block represents the constraint length (number of memory elements, i.e., in this case there is only one memory element).

\section{Yaletharatalhussein Decoding Algorithm}

The Yaletharatalhussein non-binary decoding algorithm is proposed in this paper for decoding non-binary convolutional codes. Convolutional codes differ from block codes in that a block code takes a fixed message length and encodes it, whereas a convolutional code can encode a continuous stream of data, and a hard-decision decoding can easily be realized using the Yaletharatalhusseinalgorithm. The decoder for the non-binary convolutional code finds the most probable sequence of data bits $\hat{u}$ given the received sequence $y$ :

$$
\hat{u}=\arg \{\underbrace{\max }_{u} p(u \mid y)\}
$$

where $y$ is the set of code symbols $c$ observed through noise. The above equation can be solved using the Yaletharatalhussein algorithm, explained later.

The principle states that creating a state vector con-

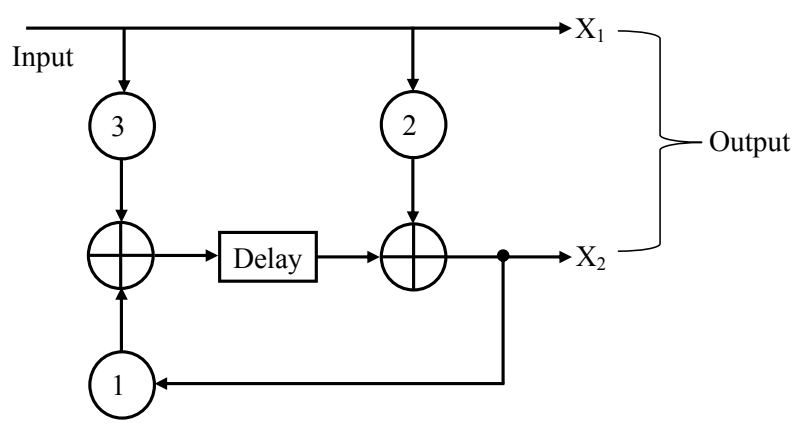

Figure 4. A 4-state ring-TCM encoder.

taining binary logic states, which represents the similarities and differences between $y$ symbols associated with each $u$ bits at the current time instant, and then searching for a minimum logic state in this vector to determine the state node number with its order bit for using in the next time instant of searching strategy method, in one hand, and for recovering the transmitted code word in the other hand. In this case, the decoding method is independent on the trellis diagram representing the non-binary convolutional encoder. The $\mathbb{Z}_{4}$-ring-TCM demodulator and decoder-based Yaletharatalhussein decoding algorithm can be represented by a flowchart as shown in Figure 5 . The flow chart inside the dashed lines represents the Yaletharatalhussein decoding algorithm.

\section{7. $\mathbb{Z}_{4}$-Ring-TCM Assisted STBC-OFDM System Overview}

$\mathbb{Z}_{4}$-Ring-TCM channel code can be combined with the $G_{2}$ STBC to improve the system performance. Figure 6 shows a schematic of the $\mathbb{Z}_{4}$-ring-TCM-assisted $G_{2}$ STBC-OFDM system. The source information bits are first encoded and modulated by the $\mathbb{Z}_{4}$-ring-TCM encoder followed by the space-time encoder, the STBC employed was the $G_{2}$ code, which invokes two transmitter antennas, and the two space-time-coded samples are mapped to two consecutive OFDM subcarriers and OFDM modulated.

The OFDM symbols are then transmitted via the multi-path fading channel, and the received noise-contaminated symbols are forwarded to the OFDM demodulator. The recovered signal is then space-time soft decoded and the soft outputs are fed to the $\mathbb{Z}_{4}$-ring-TCMdecoder which used the Yaletharatalhussein algorithm for recovering the most likely transmitted information bits. 


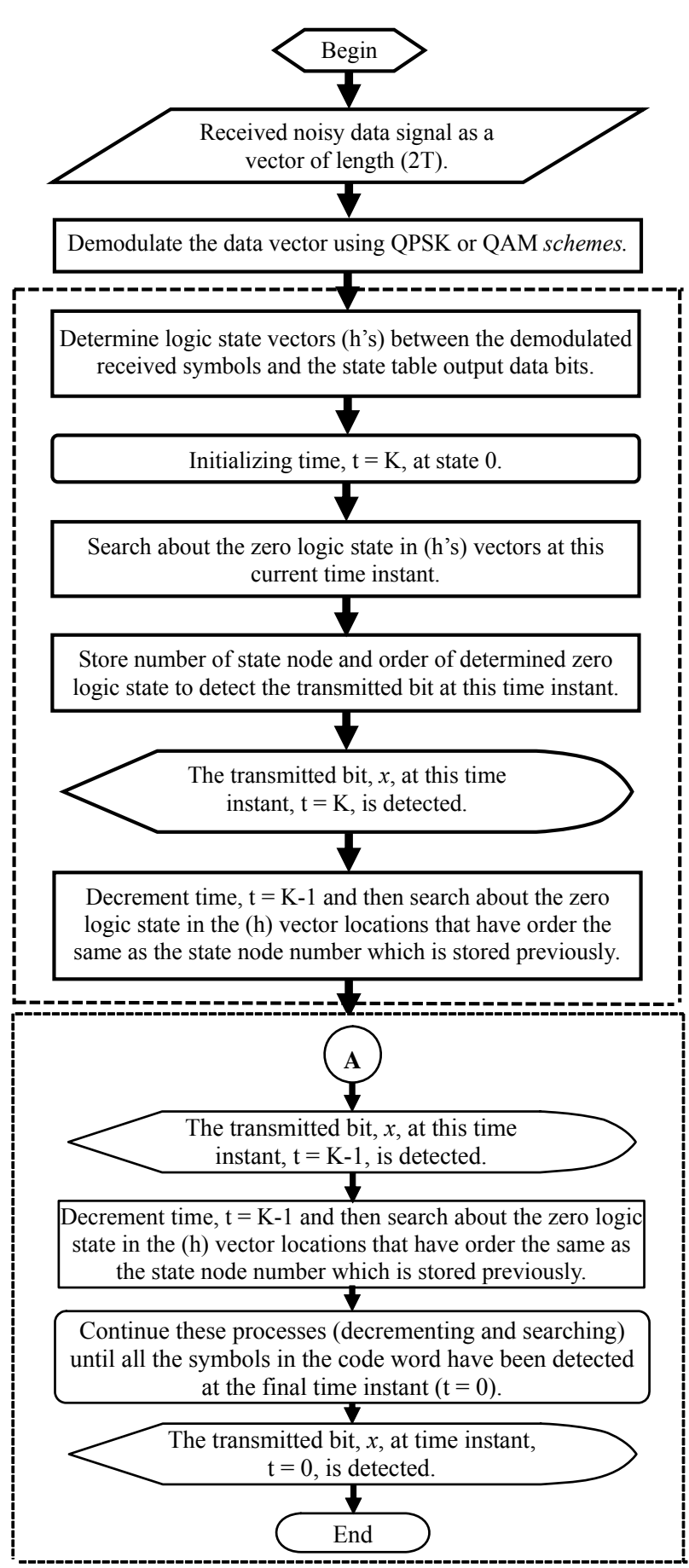

Figure 5. Flow chart of the $\mathbb{Z}_{4}$-ring-TCM demodulatordecoder-based Yaletharatalhussein decoding algorithm.

\section{Simulation Results}

The performance of the $\mathbb{Z}_{4}$-Ring-TCM-PAM-based Yaletharatalhussein decoding algorithm-assisted $G_{2}$ STBCOFDM system is studied when communicating over Rayleigh fading channel. Specifically, the short wireless asynchronous transfer mode channel impulse response [26] is considered. The simulation results can be shown

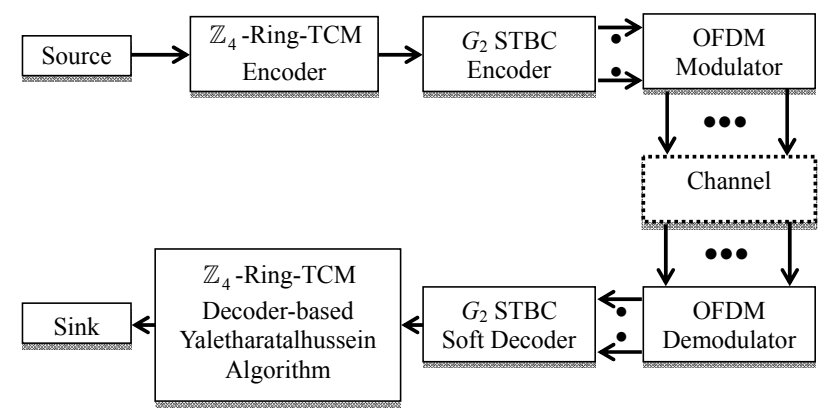

Figure 6. Schematic diagram of the proposed $\mathbb{Z}_{4}$-ringTCM-assisted $G_{2}$ STBC-OFDM system.

in Figure 7, where the (BER) versus $\left(E_{b} / N_{0}\right)$ performance is displayed in each figure including the number of symbols $(\mathrm{N})$.

For the sake of comparison with the proposed nonbinary scheme in this paper, the work [26] was studied the performance of the binary TCM-QPSK-assisted $G_{2}$ STBC-OFDM system and can be shown in Figure 8, and the performance of a 4-state ring-TCM code on urban MIMO fading channel was presented by the work [27], and can be shown in Figure 9. All the mentioned pervious TCM codes were transparent, and decoded using the soft-decision Viterbi algorithm [28,29]. The performances of the $\mathbb{Z}_{4}$-Ring-TCM scheme-based Yaletharatalhussein decoding algorithm, $\mathbb{Z}_{4}$-Ring-TCM-based Yaletharatalhussein decoding algorithm-assisted $G_{2}$ STBCOFDM scheme, and the presented schemes of works [26] and [27] can be summarized in Table 3, where the coding gains are defined as the $\left(E_{b} / N_{o}\right)$ difference, expressed in decibels, at BERs of $10^{-5}$ and $10^{-3}$ between the various channel coding assisted STBC-OFDM systems and the uncoded single transmitter system having the same effective throughput.

The performance of the best scheme in Table 3 is (printed in bold), since the performance comparison shows that the $\mathbb{Z}_{4}$-ring-TCM-PAM scheme-based Yaletharatalhussein algorithm outperforms both the $\mathbb{Z}_{4}$-ringTCM-QAM scheme and the $\mathbb{Z}_{4}$-ring-TCM-PSK schemebased Yaletharatalhussein algorithm, since this scheme is provided that the gains in $\left(E_{b} / N_{o}\right)$ are $(17.61 \mathrm{~dB})$ and $(34.82 \mathrm{~dB})$ at the BERs of $10^{-3}$ and $10^{-5}$ respectively.

Also, the $\mathbb{Z}_{4}$-ring-TCM-PAM scheme-based Yaletharatalhussein algorithm outperforms the 4-state ringTCM-based Viterbi algorithm by the gains $(8.7 \mathrm{~dB}$ and $5.5 \mathrm{~dB})$ at the BERs $\left(10^{-3}\right.$ and $\left.10^{-5}\right)$ respectively.

For the $G_{2}$ STBC-OFDM concatenated systems, the $\mathbb{Z}_{4}$-Ring-TCM-4-PAM-based Yaletharatalhussein decoding algorithm-assisted $G_{2}$ STBC-OFDM scheme outperforms the binary TCM-based Viterbi algorithm-assisted $G_{2}$ STBC-OFDM scheme by the gains; (10.93 dB and $19.87 \mathrm{~dB})$ at the BERs $\left(10^{-3}\right.$ and $\left.10^{-5}\right)$ respectively. 


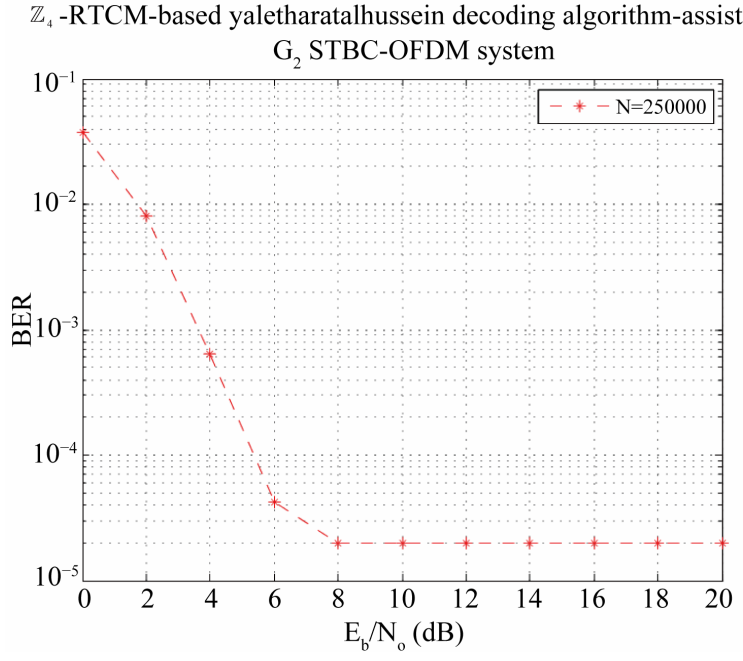

$\mathbb{Z}_{4}$-RTCM-based yaletharatalhussein decoding algorithm-assisted

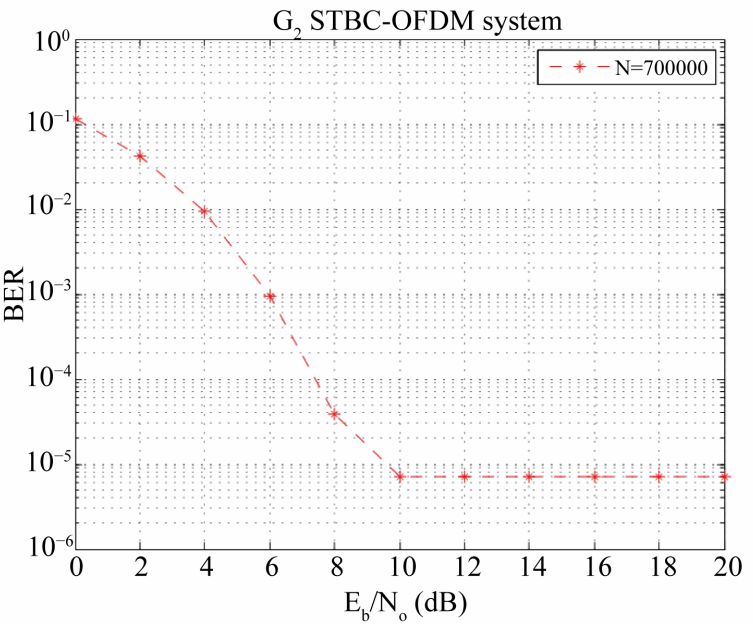

$\mathbb{Z}_{4}$-RTCM-based yaletharatalhussein decoding algorithm-assisted

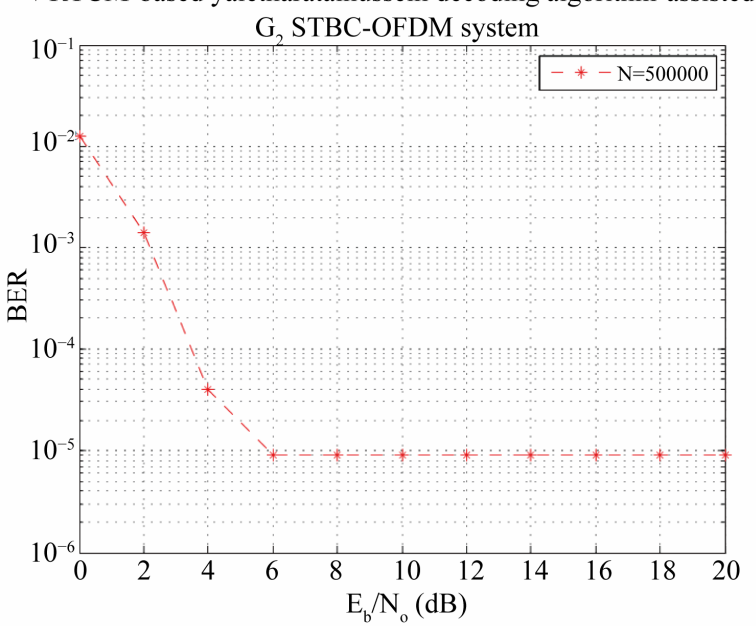

$\mathbb{Z}_{4}$-RTCM-based yaletharatalhussein decoding algorithm-assisted $\mathrm{G}_{2}$ STBC-OFDM system

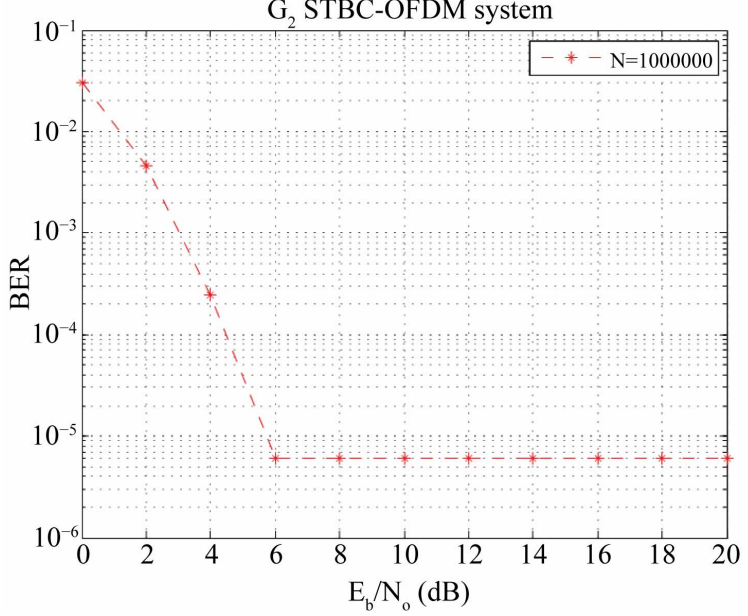

Figure 7. The (BER) versus $\left(E_{b} / N_{o}\right)$ performance of the $\mathbb{Z}_{4}$-ring-TCM-based Yaletharatalhussein decoding algorithm-assisted $G_{2}$ STBC-OFDM system.

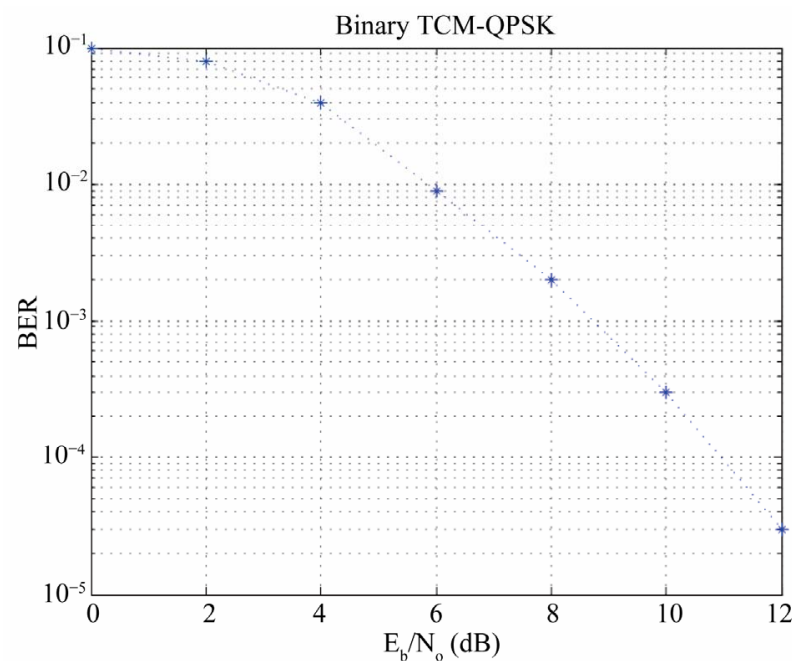

Figure 8. The (BER) versus $\left(E_{b} / N_{o}\right)$ performance of a binary TCM-QPSK-assisted $G_{2}$ STBC-OFDM system [26].

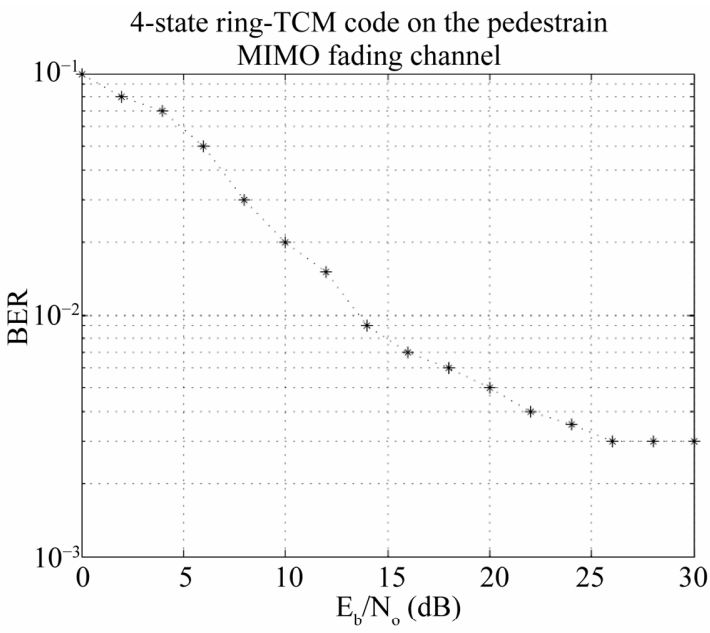

Figure 9. The (BER) versus $\left(E_{b} / N_{o}\right)$ performance of a 4 -state ring-TCM code on the pedestrian MIMO fading channel [28]. 
Table 3. Performance of the RTCM-STBC concatenated OFDM schemes.

\begin{tabular}{|c|c|c|c|c|c|c|c|}
\hline \multirow[b]{3}{*}{ STBC scheme } & \multirow[b]{3}{*}{ CM scheme } & \multirow[b]{3}{*}{ CM Code rate } & \multicolumn{4}{|c|}{ BER } & \multirow{3}{*}{$\begin{array}{c}\text { Rayleigh fading } \\
\text { Modem }\end{array}$} \\
\hline & & & \multicolumn{2}{|c|}{$E_{b} / N_{o}(\mathrm{~dB})$} & \multicolumn{2}{|c|}{ Gain (dB) } & \\
\hline & & & $10^{-3}$ & $10^{-5}$ & $10^{-3}$ & $10^{-5}$ & \\
\hline Uncoded & - & - & 24.11 & 44.12 & 0.00 & 0.00 & BPSK \\
\hline$G_{2}$ & - & - & 13.93 & 25.87 & 10.18 & 18.25 & BPSK \\
\hline- & $\begin{array}{c}\mathbb{Z}_{4} \text {-RTCM-based Yaletharatalhussein } \\
\text { algorithm }\end{array}$ & $1 / 2$ & 6.5 & 9.3 & 17.61 & 34.82 & 4-PAM \\
\hline- & 4-state RTCM-based Viterbi algorithm & $1 / 2$ & 12 & 18 & 12.11 & 26.12 & QPSK \\
\hline$G_{2}$ STBC-OFDM & Binary TCM-based Viterbi algorithm & $1 / 2$ & 8.38 & 12.44 & 5.55 & 13.43 & QPSK \\
\hline$G_{2}$ STBC-OFDM & $\begin{array}{c}\mathbb{Z}_{4} \text {-RTCM-based Yaletharatalhussein } \\
\text { algorithm }\end{array}$ & $1 / 2$ & 3 & 6 & 10.93 & 19.87 & 4-PAM \\
\hline
\end{tabular}

The performance of a 4-state ring-TCM code on the pedestrian MIMO fading channel which is shown in Figure 9 performs poorly with an error floor and thus, the $\mathbb{Z}_{4}$-Ring-TCM-4-PAM-based Yaletharatalhussein decoding algorithm-assisted $G_{2}$ STBC-OFDM scheme is the best scheme among the evaluated binary and the nonbinary schemes.

The effective throughput of the $\mathbb{Z}_{p}$-ring-TCM-PAM can be expressed by:

$$
\eta=R\left(\sqrt{p} \cdot \log _{2} M\right)
$$

where $R$, is the code rate, $p \in \mathbb{Z}$, and $M$, is the modulation order. Since, for $R=1 / 2, p=4$, and $M=4$, then $\eta=$ (2) $\mathrm{bits} / \mathrm{s} / \mathrm{Hz}$.

\section{Conclusions and Future Works}

A novel non-binary decoding method, is called Yaletharatalhussein decoding algorithm that is proposed for decoding non-binary convolutional and TCM codes, which is independent on the trellis diagram representing the non-binary convolutional encoder, as in Viterbi algorithm.

The Yaletharatalhussein algorithm employed a harddecision decoding, which needed less computational complexity over the soft-decision MLD of Viterbi algorithm. In Yaletharatalhussein algorithm, the code words are detected instantaneously through searching in the developed state vectors, while in Viterbi algorithm, the hamming distances between numbers and transition metrics are calculated and a comparison between competitive accumulated metrics is done for every state of the trellis diagram.

It is shown that the idea of TCM has been extended for symbols defined over rings of integers $\mathbb{Z}_{q}$ and that increased the bandwidth efficiency by a factor of $\sqrt{q}$ over the binary TCM code. The use of non-binary TCM led to reduction in the effective input block length, since $m$ bits of binary information correspond to one non-binary symbol for $q=2^{m}$, and thus non-binary system can be used with a high number of symbols.

The Yaletharatalhussein decoding algorithm achieved high spectral efficiency and good error performance of non-binary schemes-assisted $G_{2}$ STBC-OFDM systems, thus it can satisfy requirements of the $4 \mathrm{G}$ wireless systems over the non-binary TTCM systems due to very high computational complexity.

A future work can be done by studying the performance of the Yaletharatalhussein decoding algorithm for non-binary schemes that assisted each type of space-time coding, such as space-time trellis coding (STTC)-aided OFDM systems, and also, by studying the performance with each number of transmitters and receivers.

\section{REFERENCES}

[1] H. H. Wang, L. P. Kondi, A. Luthra and S. Ci, "4G Wireless Vedio Communications," John Wiley \& Sons Ltd., UK, 2009.

[2] S. Hara and R. Prasad, "Multicarrier Techniques for 4G Mobile Communications," Artech House, Boston, 2003.

[3] L. Hanzo and B. Choi, "Near-Instantaneously Adaptive HSDPA-Style OFDM and MC-CDMA Transceivers for WiFi, WiMAX and Next-Generation Systems," Proceedings of the IEEE, Vol. 95, No. 12, 2007, pp. 2368-2392. http://dx.doi.org/10.1109/JPROC.2007.904445

[4] K. Fazel and S. Kaiser, "Multi-Carrier and Spread Spectrum Systems from OFDM and MC-CDMA to LTE and WiMAX. John Wiley and Sons, Ltd., 2008. http://dx.doi.org/10.1002/9780470714249

[5] C. Berrou and A. Glavieux, "Near Optimum Error Correcting Coding and Decoding: Turbo Codes," IEEE Transactions on Communications, Vol. 44, No. 10, 1996, pp. 1261-1271. http://dx.doi.org/10.1109/26.539767

[6] R. Gallager, "Low Density Parity Check Codes," IEEE Transactions on Information Theory, Vol. 8, No. 1, 1962, pp. 21-28. http://dx.doi.org/10.1109/TIT.1962.1057683

[7] D. J. C. Mackay and R. M. Neal, "Near Shannon Limit Performance of Low Density Parity Check Codes," Electronics Letters, Vol. 33, No. 6, 997, pp. 457-458. http://dx.doi.org/10.1049/el:19970362 
[8] B. Lu, X. Wang and K. R. Narayanan, "LDPC-Based Space-Time Coded OFDM Systems over Correlated Fading Channels: Performance Analysis and Receiver Design," Proceedings of the 2001 IEEE International Symposium on Information Theory, Washington DC, 24-29 June 2001, p. 313.

[9] "Using MIMO-OFDM Technology to Boost Wireless LAN Performance Today," White Paper, Datacomm Research Company, St. Louis, USA, 2005.

[10] H. Sampath, S. Talwar, J. Tellado, V. Erceg and A. J. Paulraj, "A Fourth-Generation MIMO-OFDM Broadband Wireless System: Design, Performance, and field Trial Results," IEEE Communications Magazine, Vol. 40, No. 9, 2002, pp. 143-149. http://dx.doi.org/10.1109/MCOM.2002.1031841

[11] L. R. Bahl, J. Cocke, F. Jelinek and J. Raviv, "Optimal Decoding of Linear Codes for Minimizing Symbol Error Rate," IEEE Transactions on Information Theory, Vol. 20, No. 2, 1974, pp. 284-287. http://dx.doi.org/10.1109/TIT.1974.1055186

[12] J. Jayakumari, "MIMO-OFDM for 4G Wireless Systems," International Journal of Engineering Science and Technology, Vol. 2, No. 7, 2010, pp. 2886-2889.

[13] L. Hanzo, Y. (Jos) Akhtman, L. Wang and M. Jiang, "MIMO-OFDM for LTE, Wi-Fi and WiMAX Coherent versus Non-Coherent and Cooperative Turbo-Transceivers," John Wiley \& Sons Ltd., UK, 2011.

[14] Institute of Electrical and Electronics Engineers, "IEEE Standard 802.11: Wireless LAN Medium Access Control (MAC) and Physical Layer (PHY) Specifications," 18 November 1997.

[15] L. Lin, L. J. Cimini Jr. and C.-I. Chuang, "Comparison of Convolutional and Turbo Codes for OFDM with Antenna Diversity in High-Bit-Rate Wireless Applications," IEEE Communications Letters, Vol. 4, No. 9, 2000, pp. $277-$ 279.

[16] WiMAX Forum, "WiMAX Forum WiMAX Technology Forecast (2007-2012)," June 2008. http://www.wimaxforum.org/technology/downloads/

[17] A. R. S. Bahai, B. R. Saltzberg and M. Ergen, "MultiCarrier Digital Communications Theory and Applications of OFDM," 2nd Edition, Springer Science + Business Media, Inc., Boston, 2004.

[18] European Telecommunications Standards Institute, "Digital Video Broadcasting (DVB); Framing Structure, Channel Coding and Modulation for Digital Terrestrial Television (DVB-T)," ETSI ETS 300 744, March 1997.
[19] European Telecommunications Standards Institute, "Radio Equipment and Systems (RES); High Performance Radio Local Area Network (HIPERLAN) Type 1; Functional Specification," ETSI ETS 300 652, October 1996.

[20] G. Chen and X. Dong, "From Chaos to Order: Methodologies, Perspectives and Applications," World Scientific Publishing Co. Pte. Ltd., Singapore, 1998, pp. 598-614.

[21] G. Bauch, "Concatenation of Space-Time Block Codes and Turbo-TCM," Proceedings of IEEE International Conference on Communications, Vancouver, 6-10 June 1999, pp. 1202-1206.

[22] C. Berrou, A. Glavieux and P. Thitimajshima, "Near Shannon Limit Error-Correcting Coding and Decoding: Turbo Codes," Proceedings of the International Conference on Communications, Geneva, 23-26 May 1993, pp. 1064-1070.

[23] P. Robertson and T. Worz, "Bandwidth Efficient Turbo Trellis-Coded Modulation Using Punctured Component Codes," IEEE Journal on Selected Areas in Communications, Vol. 16, No. 2, 1998, pp. 206-218. http://dx.doi.org/10.1109/49.661109

[24] R. Carrasco, F. Lopez and P. Farrell, "Ring-TCM for M-PSK Modulation: AWGN Channels and DSP Implementation," IEE Proceedings-Communications, Vol. 143, No. 5, 1996, pp. 273-280.

[25] E. H. Connel, "Elements of Abstract and Linear Algebra," Coral Gables, 2004.

[26] L. Hanzo, T. H. Liew and B. L. Yeap, "Turbo Coding, Turbo Equalisation and Space-Time Coding," IEEE Press and John Wiley \& Sons, Ltd., Chichester, 2002. http://www-mobile.ecs.soton.ac.uk

[27] A. J. Paulraj, D. A. Gore, R. U. Nabar and H. Bolcskei, "An Overview of MIMO Communications-A Key to Gigabit Wireless," Proceedings of the IEEE, Vol. 92, No. 2, 2004, pp. 198-218. http://dx.doi.org/10.1109/JPROC.2003.821915

[28] A. J. Viterbi, "Error Bounds for Convolutional Codes and Asymptotically Optimum Decoding Algorithm," IEEE Transactions on Information Theory, Vol. 13, No. 2, 1967, pp. 260-269. http://dx.doi.org/10.1109/TIT.1967.1054010

[29] G. D. Forney, "The Viterbi Algorithm," Proceedings of IEEE, Vol. 61, No. 3, 1973, pp. 268-278. http://dx.doi.org/10.1109/PROC.1973.9030 